\title{
Data report: pore water nitrate and silicate concentrations for Expedition 320/321 Pacific Equatorial Age Transect ${ }^{1}$
}

\author{
Wendy E.C. Kordesch ${ }^{2,3}$ and Margaret L. Delaney ${ }^{2}$
}

\section{Chapter contents}

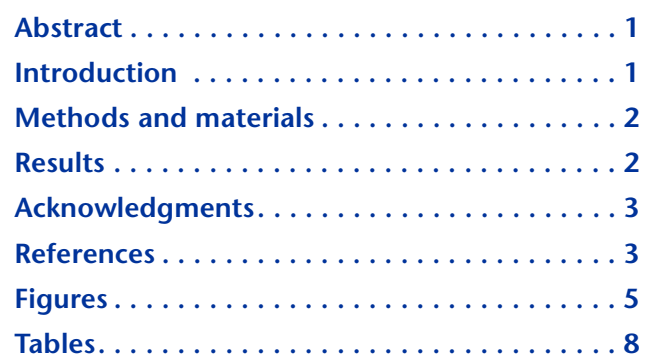

${ }^{1}$ Kordesch, W.E.C., and Delaney, M.L., 2013. Data report: pore water nitrate and silicate concentrations for Expedition 320/321 Pacific Equatorial Age Transect. In Pälike, H., Lyle, M., Nishi, H., Raffi, I., Gamage, K., Klaus, A., and the Expedition 320/321 Scientists, Proc. IODP, 320/ 321: Tokyo (Integrated Ocean Drilling Program Management International, Inc.). doi:10.2204/iodp.proc.320321.210.2013 2Ocean Sciences Department, Institute of Marine Sciences, University of California Santa Cruz, 1156 High Street, Santa Cruz CA 95064, USA. ${ }^{3}$ Present address: School of Ocean and Earth Science, National Oceanography Centre, University of Southampton, European Way, Southampton SO14 3ZH, United Kingdom. wendy.kordesch@noc.soton.ac.uk

\section{Abstract}

Concentrations of dissolved nitrate and silicate were determined in pore water samples from all eight sites (U1331-U1338) of the Pacific Equatorial Age Transect, Integrated Ocean Drilling Program (IODP) Expedition 320/321. Nitrate measurements were not made shipboard, and they are useful in assessing the extent of suboxic diagenesis evident in these sites. Dissolved silicate concentrations were remeasured in this study because two different analytical techniques were used shipboard during Expeditions $320 / 321$, and comparisons indicated issues with analytical consistency. Nitrate and silicate were measured simultaneously in pore water samples squeezed from whole rounds and obtained by Rhizon sampling using an automated colorimetric technique with flow injection analysis. Profiles of nitrate concentrations at Sites U1331-U1333 show the least variability with depth $(\sim 50-80 \mu \mathrm{M})$. Sites U1334-U1336 exhibit pronounced depletions (from $\sim 300$ to $0 \mu \mathrm{M}$ at Site U1334, from $\sim 150$ to $1 \mu \mathrm{M}$ at Site U1335, and from $\sim 75$ to $10 \mu \mathrm{M}$ at Site U1336), whereas nitrate concentrations increase with depth at Sites U1337 and U1338 (to 64 and $198 \mu \mathrm{M}$, respectively). Silicate concentrations at Site U1331 reach $\sim 1180$ $\mu \mathrm{M}$ at $\sim 8$ meters below seafloor, whereas profiles at other sites generally increase with depth (from 550 to $1200 \mu \mathrm{M}$ at Site U1332, from 700 to $1200 \mu \mathrm{M}$ at Site U1333, from 800 to $1350 \mu \mathrm{M}$ at Site U1334, from 870 to $1960 \mu \mathrm{M}$ at Site U1337, and from 730 to $1560 \mu \mathrm{M}$ at Site U1338). At Sites U1335 and U1336, silicate varies 800-1220 and 760-1100 $\mu \mathrm{M}$, respectively.

\section{Introduction}

During Integrated Ocean Drilling Program (IODP) Expedition $320 / 321$, pore water samples were obtained from eight sites (U1331-U1338; Table T1) in the equatorial Pacific. These expeditions were the first after the extensive refit and laboratory updating for the R/V JOIDES Resolution completed in early 2009. Pore water measurements previously done with long-established techniques using an ultraviolet-visible light spectrophotometer (Gieskes et al., 1991) were to be carried out with newly adapted techniques using a discrete analyzer (see the "Methods" chapter [Expedition 320/321 Scientists, 2010a]). These techniques had not been thoroughly vetted before the expeditions, the module needed for nitrate measurements was not present/working during 
Expedition 320, and the discrete analyzer failed entirely before Expedition 321. As a result, nitrate measurements were not made shipboard, and silicate measurements were made using two different analytical techniques. To address these gaps, we report the concentrations of dissolved nitrate and silicate measured at a shore-based facility from archived splits of pore water samples obtained by whole-round squeezing and from Rhizon sampling.

The quantification of nitrate concentrations complements shipboard pore water measurements of dissolved manganese, iron, and sulfate (see the "Expedition 320/321 summary" chapter [Pälike et al., $2010 b]$ ) in the characterization of diagenetic processes involving organic carbon. Our shore-based measurements of dissolved silicate were prompted by inconsistencies (up to $\sim 200 \mu \mathrm{M}$ ) (Expedition 320/ 321 Scientists, 2010a) between repeated shipboard analyses of reference samples caused by the use of two different analytical techniques during Expeditions 320 and 321. Expedition 320 used an OI Analytical discrete analyzer (DA3500) spectrophotometer, which failed before Expedition 321, and inductively coupled plasma-atomic emission spectroscopy was used in its place (Expedition 320/321 Scientists, 2010a). For analytical continuity, we measured all archived pore water samples using an automated coulometric technique in a flow injection analysis system (Lachat QuickChem 8000 FIA).

Measurements were made on two archived pore water sample types: whole round and Rhizon. Wholeround samples are hydraulically squeezed from 5-10 $\mathrm{cm}$ long whole-round sediment specimens and are a routine part of geochemical measurements on IODP expeditions. Whole-round sample resolution is generally one sample per section for all sites. Rhizon sampling uses plastic syringes to pull pore water through thin tubes inserted directly into core liners. This type of sampling was adapted for measurement of pore water in sediment during IODP Expedition 302 (Dickens et al., 2007) and provides a nondestructive method to obtain higher resolution sampling. Sites U1331, U1334, U1337, and U1338 include Rhizon samples (up to every $10 \mathrm{~cm}$ at Sites U1331 and U1338 and every $\sim 1 \mathrm{~m}$ at other sites) across zones where pronounced chemical gradients and sediment color changes occur.

\section{Methods and materials}

Archived pore water whole-round and Rhizon samples collected during Expedition 320/321 were analyzed simultaneously for dissolved nitrate and silicate concentrations. Samples were filtered and sealed in glass ampules during Expedition 320/321. Am- pules were opened on the day of analysis. Samples and standards were diluted with glass-distilled water (6:1 for nitrate and 20:1 for silicate), and constituents were measured using an automated coulometric technique in a flow injection analysis system (Lachat QuickChem 8000 FIA) using standard methods (Parsons et al., 1984; Strickland and Parsons, 1968). When sample size was limited, measurements of nitrate were prioritized.

Standards were made from a stock solution of potassium nitrate, sodium fluorosilicate, and glass-distilled water. A consistency standard (a mixture of sodium nitrate, sodium fluorosilicate, and sodium chloride dissolved in glass-distilled water and kept under refrigeration) was processed and analyzed with each batch of samples for long-term calibration and quality control purposes. Analytical error on the consistency standard analyzed in multiple replicates $(n=57)$ over the course of several months was $48.6 \pm$ $3.4 \mu \mathrm{M}$ for nitrate and $722 \pm 12 \mu \mathrm{M}$ for silicate (Table T2). Detection limits, determined from replicates ( $n$ $=10$ ) of the glass-distilled water used for dilution and run as samples were $0.7 \pm 0.2 \mu \mathrm{M}$ for nitrate and $1 \pm 0.4 \mu \mathrm{M}$ for silicate (Table T2).

\section{Results}

Concentrations of dissolved nitrate and silicate for all eight Expedition 320/321 sites are provided in Tables T3, T4, T5, T6, T7, T8, T9, and T10 and Figures F1 and F2. Depths are plotted in core depth below seafloor, method A (meters below seafloor [mbsf] in text and figures), for all sites.

The profile of dissolved nitrate concentrations from our measurements on whole-round and Rhizon samples at Site U1331 shows a slight decrease with depth, starting at $\sim 70 \mu \mathrm{M}$ at 2.95 mbsf and decreasing to an average of $\sim 55 \mu \mathrm{M}$ at depths greater than 22 mbsf. At Site U1332, concentrations remain between $\sim 55$ and $45 \mu \mathrm{M}$. Nitrate concentrations at Site U1333 range from 50 to $60 \mu \mathrm{M}$ and increase to 83 $\mu \mathrm{M}$ at 35.95 mbsf. Site U1334 contains the highest nitrate measured at any site, with concentrations in the shallowest sample at $\sim 261 \mu \mathrm{M}$ followed by a gradual decline from $\sim 44 \mu \mathrm{M}$ at 6 mbsf to $\sim 10 \mu \mathrm{M}$ at 125 mbsf and a slight increase to $\sim 50 \mu \mathrm{M}$ at 228 mbsf. Site U1335 follows a similar profile, with high nitrate in the shallowest samples $(\sim 153 \mu \mathrm{M})$, a sharp decline to near-zero values (54-375 mbsf), and a slight increase at depth $(\sim 34 \mu \mathrm{M})$. Near-surface concentrations at Site U1336 start at $\sim 75 \mu \mathrm{M}$ and steadily decrease to $\sim 10 \mu \mathrm{M}$ by $93 \mathrm{mbsf}$. At Site U1337, nitrate remains below $30 \mu \mathrm{M}$ from 7 to 409 mbsf and increases to $\sim 64 \mu \mathrm{M}$ at $\sim 428 \mathrm{mbsf}$. Site U1338 also remains low $(<75 \mu \mathrm{M})$ from 1 to 350 
mbsf and increases at $360 \mathrm{mbsf}$ to $200 \mu \mathrm{M}$. Deepwater nitrate concentrations from the World Ocean Circulation Experiment Pacific Ocean Atlas in the equatorial Pacific (Line P04; $10^{\circ} \mathrm{N},-120^{\circ} \mathrm{W} ; \sim 4500 \mathrm{~m}$ water depth) proximal to Expedition 320/321 sites are steady at $\sim 36 \mu \mathrm{M}$ (Talley, 2007).

Profiles of dissolved silicate concentrations from our measurements on whole-round and Rhizon samples at most sites increase with depth. At Site U1331, surface concentrations of $\sim 750 \mu \mathrm{M}$ at $3 \mathrm{mbsf}$ approach $900 \mu \mathrm{M}$ at 123 mbsf. Surface concentrations at Site U1332 contain the lowest measured silicate concentrations of $\sim 555 \mu \mathrm{M}$ at $3 \mathrm{mbsf}$ and approach 1200 $\mu \mathrm{M}$ at 103 mbsf. Profiles of silica at Sites U1333, U1335, and U1336 remain close to $\sim 1000 \mu \mathrm{M}$. Silicate at Site U1334 increases from $\sim 900 \mu \mathrm{M}$ at shallow depths to $1362 \mu \mathrm{M}$ at $257 \mathrm{mbsf}$. Silicate at both Sites U1337 and U1338 increases from $\sim 1000 \mu \mathrm{M}$ to the highest measured values of more than $\sim 1500 \mu \mathrm{M}$. Rhizon sampling at Site U1338 also targeted a diatom-rich interval at 130 mbsf (Section 321-U1333B$14 \mathrm{H}-5$; see the "Site U1338" chapter [Expedition 320/321 Scientists, 2010e]) and contains average silicate concentrations of $\sim 1150 \mu \mathrm{M}$.

Silicate concentrations measured in this study (including Rhizon samples) are consistently higher than shipboard measurements from Expedition 320/ $321\left(0.67 \mu \mathrm{M} / \mu \mathrm{M} ; R^{2}=0.41 ; n=380\right)$, falling below a 1:1 line (Fig. F3). When compared by expedition, our data are on average more similar to shipboard measurements made during Expedition 321 (Sites U1337 and U1338; $\left.0.56 \mu \mathrm{M} / \mu \mathrm{M} ; R^{2}=0.40 ; n=153\right)$ than during Expedition 320 (Sites U1331-U1336; 0.19 $\mu \mathrm{M} / \mu \mathrm{M} ; R^{2}=0.07 ; n=227$ ).

Rhizon samples from a $1.3 \mathrm{~m}$ interval at Site U1331 (Section 320-U1331B-1H-6; Table T3) were collected to test the feasibility of using the Rhizon sampling procedure during Expedition 320/321. Shipboard measurements and our archived samples show general agreement between whole-round and Rhizon sample concentrations (Table T3; Figs. F1, F2) (Expedition 320/321 Scientists, 2010b).

Differences in concentrations between whole-round and Rhizon samples at Sites U1331 and U1334 are partially attributed to samples originating from different holes (i.e., whole-round samples at Site U1331 are from Hole U1331A and Rhizon samples are from Hole U1331B; Table T3). Additionally, interstitial water sampling from extended core barrel-cored sections may contain contamination from seawater drilling fluid (i.e., Samples 320-U1334C-23X-1, 75 $\mathrm{cm}$, to 23X-3, $75 \mathrm{~cm}$, as well as Site U1337 below 210 mbsf [Section 321-U1337A-23X-3] and Site U1338 below 244 mbsf [Section 321-U1338A-27X-3) (Expedition 320/321 Scientists, 2010c, 2010d, 2010e).

\section{Acknowledgments}

We thank R. Franks and the IMS Marine Analytical Laboratory for analytical assistance. This research used samples and data provided by the Integrated Ocean Drilling Program (IODP). IODP is sponsored by the U.S. National Science Foundation and participating countries. Research funding to M. Delaney was provided by the U.S. Science Support Program associated with IODP managed by the Consortium for Ocean Leadership.

\section{References}

Dickens, G.R., Koelling, M., Smith, D.C., Schneiders, L., and the IODP Expedition 302 Scientists, 2007. Rhizon sampling of pore waters on scientific drilling expeditions: an example from the IODP Expedition 302, Arctic Coring Expedition (ACEX). Sci. Drill., 4:22-25.

doi:10.2204/iodp.sd.4.08.2007

Expedition 320/321 Scientists, 2010a. Methods. In Pälike, H., Lyle, M., Nishi, H., Raffi, I., Gamage, K., Klaus, A., and the Expedition 320/321 Scientists, Proc. IODP, 320/ 321: Tokyo (Integrated Ocean Drilling Program Management International, Inc.). doi:10.2204/ iodp.proc.320321.102.2010

Expedition 320/321 Scientists, 2010b. Site U1331. In Pälike, H., Lyle, M., Nishi, H., Raffi, I., Gamage, K., Klaus, A., and the Expedition 320/321 Scientists, Proc. IODP, 320/321: Tokyo (Integrated Ocean Drilling Program Management International, Inc.). doi:10.2204/ iodp.proc.320321.103.2010

Expedition 320/321 Scientists, 2010c. Site U1334. In Pälike, H., Lyle, M., Nishi, H., Raffi, I., Gamage, K., Klaus, A., and the Expedition 320/321 Scientists, Proc. IODP, 320/321: Tokyo (Integrated Ocean Drilling Program Management International, Inc.). doi:10.2204/ iodp.proc.320321.106.2010

Expedition 320/321 Scientists, 2010d. Site U1337. In Pälike, H., Lyle, M., Nishi, H., Raffi, I., Gamage, K., Klaus, A., and the Expedition 320/321 Scientists, Proc. IODP, 320/321: Tokyo (Integrated Ocean Drilling Program Management International, Inc.). doi:10.2204/ iodp.proc.320321.109.2010

Expedition 320/321 Scientists, 2010e. Site U1338. In Pälike, H., Lyle, M., Nishi, H., Raffi, I., Gamage, K., Klaus, A., and the Expedition 320/321 Scientists, Proc. IODP, 320/321: Tokyo (Integrated Ocean Drilling Program Management International, Inc.). doi:10.2204/ iodp.proc.320321.110.2010

Gieskes, J.M., Gamo, T., and Brumsack, H., 1991. Chemical methods for interstitial water analysis aboard JOIDES Resolution. ODP Tech. Note, 15. doi:10.2973/

odp.tn.15.1991 
Pälike, H., Lyle, M., Nishi, H., Raffi, I., Gamage, K., Klaus, A., and the Expedition 320/321 Scientists, 2010a. Proc. IODP, 320/321: Tokyo (Integrated Ocean Drilling Program Management International, Inc.). doi:10.2204/ iodp.proc.320321.2010

Pälike, H., Nishi, H., Lyle, M., Raffi, I., Gamage, K., Klaus, A., and the Expedition 320/321 Scientists, 2010b. Expedition 320/321 summary. In Pälike, H., Lyle, M., Nishi, H., Raffi, I., Gamage, K., Klaus, A., and the Expedition 320/321 Scientists, Proc. IODP, 320/321: Tokyo (Integrated Ocean Drilling Program Management International, Inc.). doi:10.2204/iodp.proc.320321.101.2010

Parsons, T.R., Maita, Y., and Lalli, C.M., 1984. A Manual of Chemical and Biological Methods for Seawater Analysis: Oxford, (Pergamon Press).
Strickland, J.D.H., and Parsons, T.R., 1968. A Practical Handbook of Seawater Analysis. Bull. Fish. Res. Board Can., 167.

Talley, L.D., 2007. Hydrographic Atlas of the World Ocean Circulation Experiment (WOCE) (Vol. 2): Pacific Ocean. Sparrow, M., Chapman, P., and Gould, J. (Eds.):

Southampton, U.K. (International WOCE Project Office). http://www-pord.ucsd.edu/whp_atlas/ pacific_index.html

Initial receipt: 29 June 2012

Acceptance: 17 January 2013

Publication: 16 April 2013

MS 320321-210 

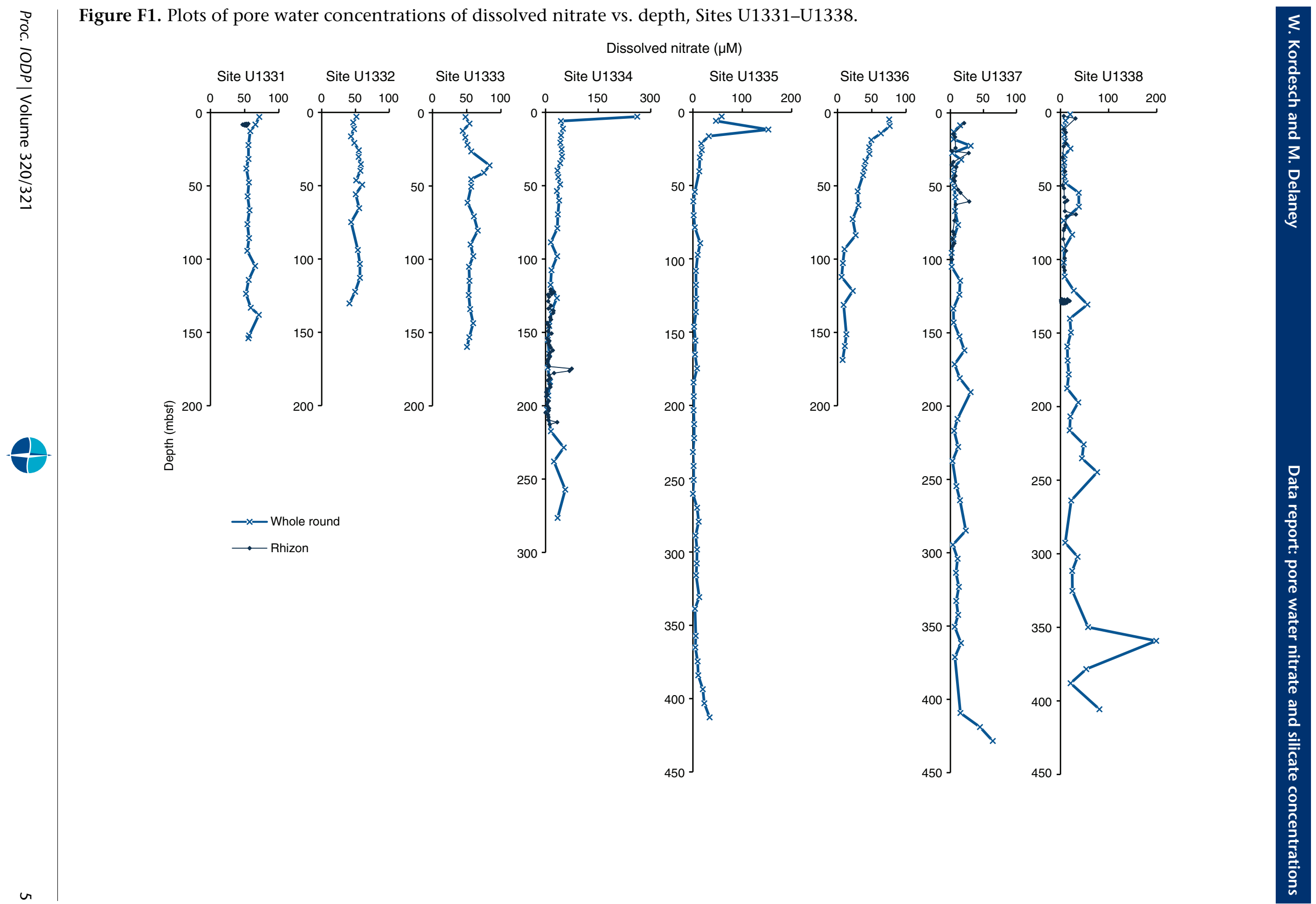
Figure F2. Plots of pore water concentrations of dissolved silicate vs. depth, Sites U1331-U1338.

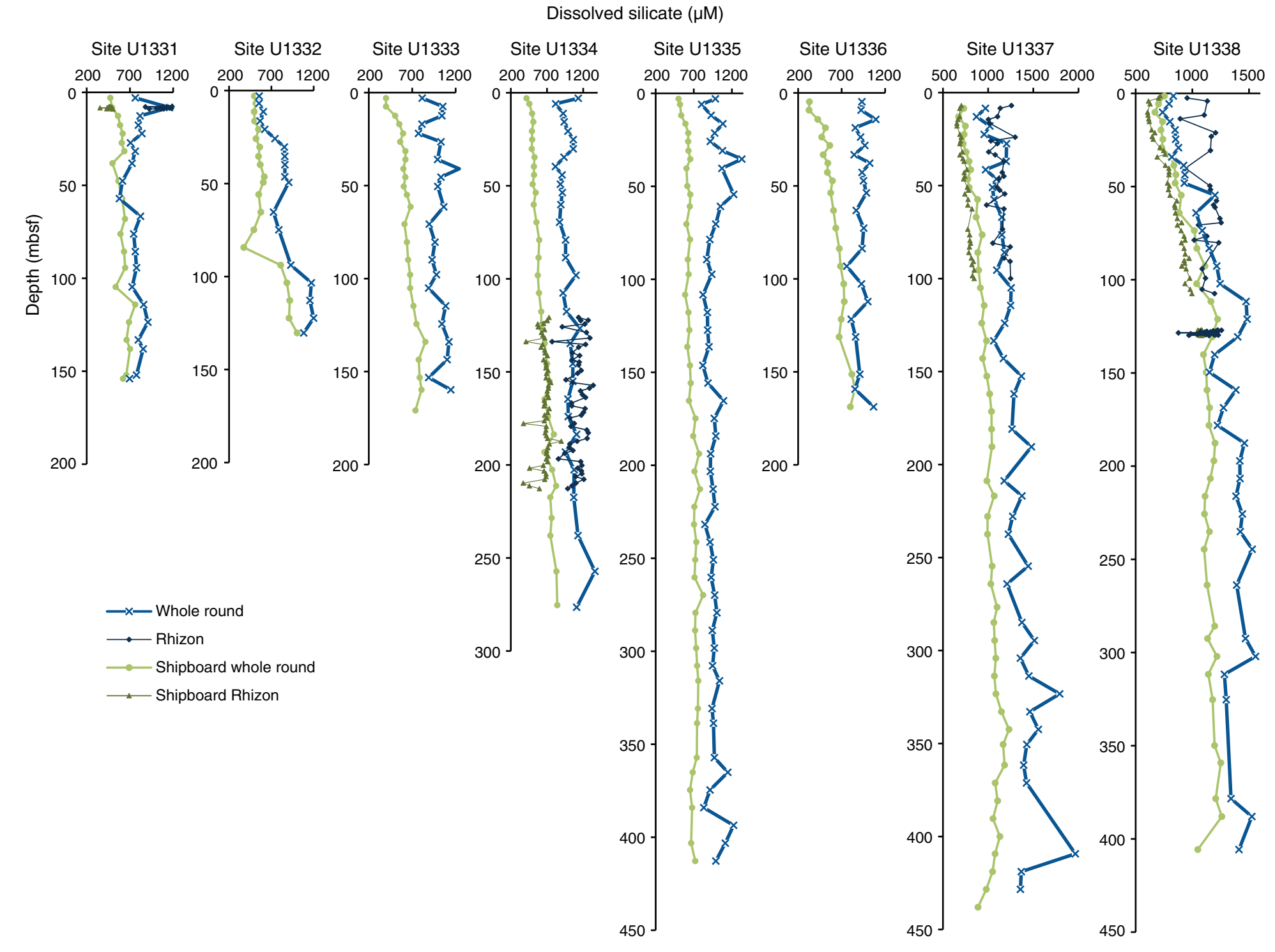


Figure F3. Comparison plot of dissolved silicate data measured during Expedition 320/321 vs. measurements made in this study. Solid lines are linear regressions for Expeditions $320\left(y=0.19 x+441 ; R^{2}=0.07 ; n=227\right)$ and $321\left(y=0.56 x+272 ; R^{2}=0.40 ; n=153\right)$. Linear regression for all data is $y=0.67 x+42\left(R^{2}=0.41 ; n=380\right)$. Dotted line is $1: 1$.

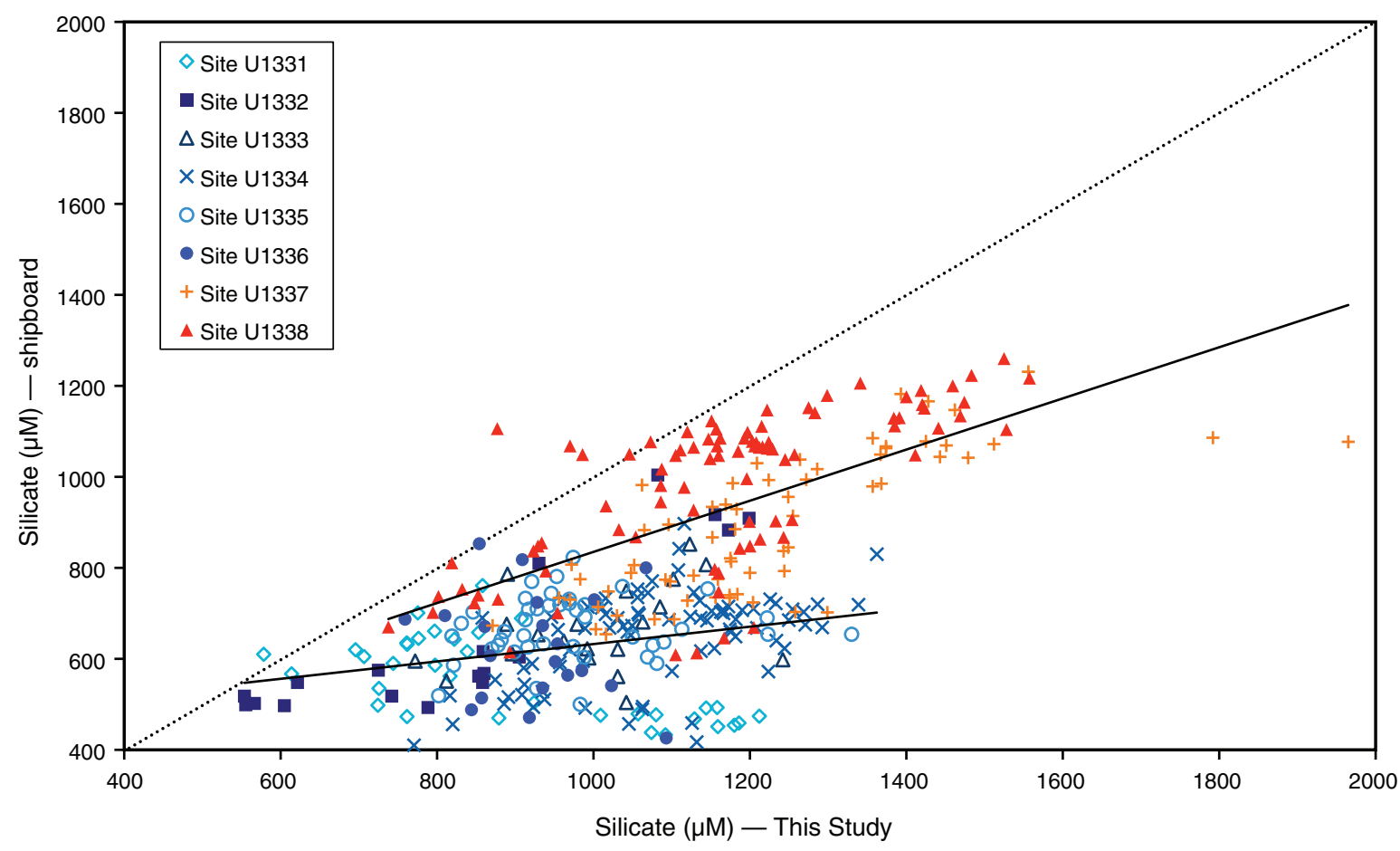


Table T1. Equatorial Pacific site information, Expedition 320/321.

\begin{tabular}{clccccc}
\hline Site & $\begin{array}{c}\text { Holes with } \\
\text { whole-round } \\
\text { samples }\end{array}$ & $\begin{array}{c}\text { Holes with } \\
\text { Rhizon } \\
\text { samples }\end{array}$ & $\begin{array}{c}\text { Water depth } \\
\text { (mbsl) }\end{array}$ & Latitude & Longitude & $\begin{array}{c}\text { Crustal age } \\
\text { (Ma) }\end{array}$ \\
\hline U1331 & A, B & B & 5116 & $12^{\circ} 04.088^{\prime} \mathrm{N}$ & $142^{\circ} 09.696^{\prime} \mathrm{W}$ & 53 \\
U1332 & A & & 4924 & $11^{\circ} 54.709^{\prime} \mathrm{N}$ & $141^{\circ} 02.742^{\prime} \mathrm{W}$ & 50 \\
U1333 & A & & 4853 & $10^{\circ} 30.995^{\prime} \mathrm{N}$ & $138^{\circ} 25.172^{\prime} \mathrm{W}$ & 46 \\
U1334 & A & B, C & 4799 & $7^{\circ} 59.998^{\prime} \mathrm{N}$ & $131^{\circ} 58.393^{\prime} \mathrm{W}$ & 38 \\
U1335 & A, B & & 4327 & $5^{\circ} 18.734^{\prime} \mathrm{N}$ & $126^{\circ} 116.994^{\prime} \mathrm{W}$ & 26 \\
U1336 & B & & 4286 & $7^{\circ} 42.073^{\prime} \mathrm{N}$ & $128^{\circ} 15.252^{\prime} \mathrm{W}$ & $\sim 33^{*}$ \\
U1337 & A & A & 4463 & $3^{\circ} 50.006^{\prime} \mathrm{N}$ & $123^{\circ} 12.355^{\prime} \mathrm{W}$ & 24 \\
U1338 & A, B & A, B & 4200 & $2^{\circ} 30.468^{\prime} \mathrm{N}$ & $117^{\circ} 58.162^{\prime} \mathrm{W}$ & 18 \\
\hline
\end{tabular}

* = drilling did not reach basement; crustal age unknown. From Pälike et al. (2010).

Table T2. Analytical figures of merit.

\begin{tabular}{lcc}
\hline & \multicolumn{2}{c}{ Pore water concentration $(\mu \mathrm{M})$} \\
\cline { 2 - 3 } & $\mathrm{NO}_{3}{ }^{-}$ & $\mathrm{H}_{4} \mathrm{SiO}_{4}{ }^{0}$ \\
\hline Detection limits & $0.7 \pm 0.2$ & $1 \pm 0.4$ \\
Reproducibility (consistency standard replicates) & $48.6 \pm 3.4$ & $722 \pm 12$ \\
\hline
\end{tabular}

Detection limits are defined as three times the standard deviation of replicate measures of a blank of the same matrix as each sample, expressed in equivalent concentration for a typical sample size. 
Table T3. Pore water concentrations, Site U1331.

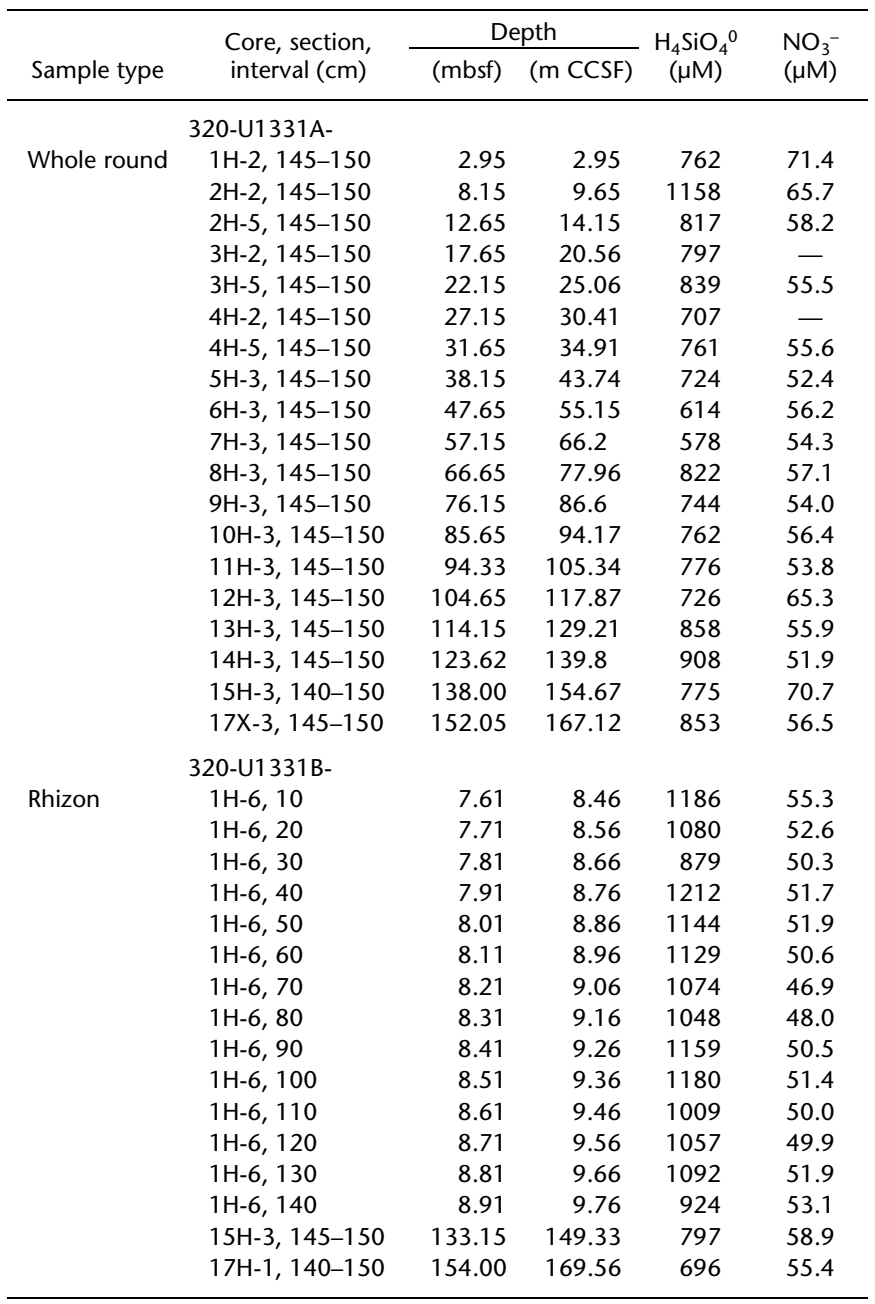

$-=$ no data. 
Table T4. Pore water concentrations, Site U1332.

\begin{tabular}{|c|c|c|c|c|c|}
\hline \multirow[b]{2}{*}{ Sample type } & \multirow{2}{*}{$\begin{array}{l}\text { Core, section, } \\
\text { interval }(\mathrm{cm})\end{array}$} & \multicolumn{2}{|c|}{ Depth } & \multirow{2}{*}{$\begin{array}{c}\mathrm{H}_{4} \mathrm{SiO}_{4}{ }^{0} \\
(\mu \mathrm{M})\end{array}$} & \multirow{2}{*}{$\begin{array}{l}\mathrm{NO}_{3} \\
(\mu \mathrm{M})\end{array}$} \\
\hline & & (mbsf) & (m CCSF) & & \\
\hline & 320-U1332A- & & & & \\
\hline \multirow{19}{*}{ Whole round } & $1 \mathrm{H}-2,145-150$ & 2.95 & 2.95 & 555 & 51.4 \\
\hline & $2 \mathrm{H}-2,145-150$ & 6.85 & 7.16 & 554 & 46.6 \\
\hline & $2 \mathrm{H}-5,145-150$ & 11.35 & 11.66 & 605 & 47.9 \\
\hline & $3 \mathrm{H}-2,145-150$ & 16.35 & 17.00 & 566 & 43.3 \\
\hline & $3 \mathrm{H}-5,145-150$ & 20.85 & 21.50 & 622 & 48.3 \\
\hline & $4 \mathrm{H}-2,145-150$ & 25.85 & 28.50 & 742 & 55.0 \\
\hline & $4 \mathrm{H}-5,145-150$ & 30.35 & 33.00 & 853 & 54.7 \\
\hline & $5 \mathrm{H}-2,145-150$ & 35.35 & 40.50 & 858 & 58.0 \\
\hline & $5 \mathrm{H}-5,145-150$ & 39.85 & 45.00 & 860 & 57.3 \\
\hline & $6 \mathrm{H}-3,145-150$ & 46.35 & 52.00 & 859 & 51.0 \\
\hline & $6 \mathrm{H}-5,145-150$ & 49.35 & 55.00 & 905 & 60.0 \\
\hline & 7H-3, 145-150 & 55.85 & 63.75 & - & 50.4 \\
\hline & $8 \mathrm{H}-3,145-150$ & 65.35 & 72.85 & 725 & 55.5 \\
\hline & $9 \mathrm{H}-3,145-150$ & 74.85 & 77.63 & 788 & 43.6 \\
\hline & $11 \mathrm{H}-3,145-150$ & 93.85 & 97.17 & 930 & 53.5 \\
\hline & $12 \mathrm{H}-3,145-150$ & 103.35 & 109.37 & 1173 & 56.6 \\
\hline & $13 \mathrm{H}-3,145-150$ & 112.85 & 120.62 & 1155 & 56.6 \\
\hline & $14 \mathrm{H}-3,145-150$ & 122.35 & 133.87 & 1199 & 49.5 \\
\hline & $15 X-3,140-150$ & 130.30 & 142.82 & 1082 & 41.1 \\
\hline
\end{tabular}

$-=$ no data.

Table T5. Pore water concentrations, Site U1333.

\begin{tabular}{ccrrr}
\hline \multirow{3}{*}{ Sample type } & $\begin{array}{c}\text { Core, section, } \\
\text { interval (cm) }\end{array}$ & $\begin{array}{c}\text { Depth } \\
(\mathrm{mbsf})\end{array}$ & $\begin{array}{c}\mathrm{H}_{4} \mathrm{SiO}_{4}{ }^{0} \\
(\mu \mathrm{M})\end{array}$ & $\begin{array}{r}\mathrm{NO}_{3}{ }^{-} \\
(\mu \mathrm{M})\end{array}$ \\
\hline 320-U1333A- & & & \\
& $1 \mathrm{H}-2,145-150$ & 2.95 & 815 & 48.2 \\
& $1 \mathrm{H}-5,145-150$ & 7.45 & 1052 & 54.1 \\
& $2 \mathrm{H}-2,145-150$ & 12.45 & 1042 & 44.2 \\
& $2 \mathrm{H}-5,145-150$ & 16.95 & 812 & 48.0 \\
& $3 \mathrm{H}-2,145-150$ & 21.95 & 772 & 51.1 \\
& $3 \mathrm{H}-5,145-150$ & 26.45 & 1031 & 56.6 \\
& $4 \mathrm{H}-5,145-150$ & 35.95 & 992 & 83.4 \\
& $5 \mathrm{H}-2,145-150$ & 40.95 & 1242 & 75.2 \\
& $5 \mathrm{H}-5,145-150$ & 45.45 & 1031 & 56.5 \\
& $6 \mathrm{H}-2,145-150$ & 50.45 & 994 & 56.5 \\
& $7 \mathrm{H}-3,140-150$ & 61.40 & 1063 & 51.2 \\
& $8 \mathrm{H}-3,140-150$ & 70.70 & 896 & 60.5 \\
& $9 \mathrm{H}-3,140-150$ & 80.40 & 962 & 66.2 \\
& $10 \mathrm{H}-3,140-150$ & 89.90 & 929 & 55.6 \\
& $11 \mathrm{X}-2,140-150$ & 97.90 & 979 & 59.6 \\
& $12 \mathrm{X}-3,140-150$ & 105.10 & 889 & 53.3 \\
$13 \mathrm{X}-3,140-150$ & 114.70 & 1085 & 54.0 \\
& $14 \mathrm{X}-3,140-150$ & 124.40 & 1042 & 52.9 \\
$15 \mathrm{X}-3,140-150$ & 134.00 & 1123 & 55.0 \\
$16 \mathrm{X}-3,140-150$ & 143.60 & 1102 & 59.4 \\
$17 \mathrm{X}-3,140-150$ & 153.20 & 890 & 53.8 \\
$18 \mathrm{X}-1,140-150$ & 159.80 & 1144 & 50.4 \\
\hline & & & &
\end{tabular}


Table T6. Pore water concentrations, Site U1334. (Continued on next page.)

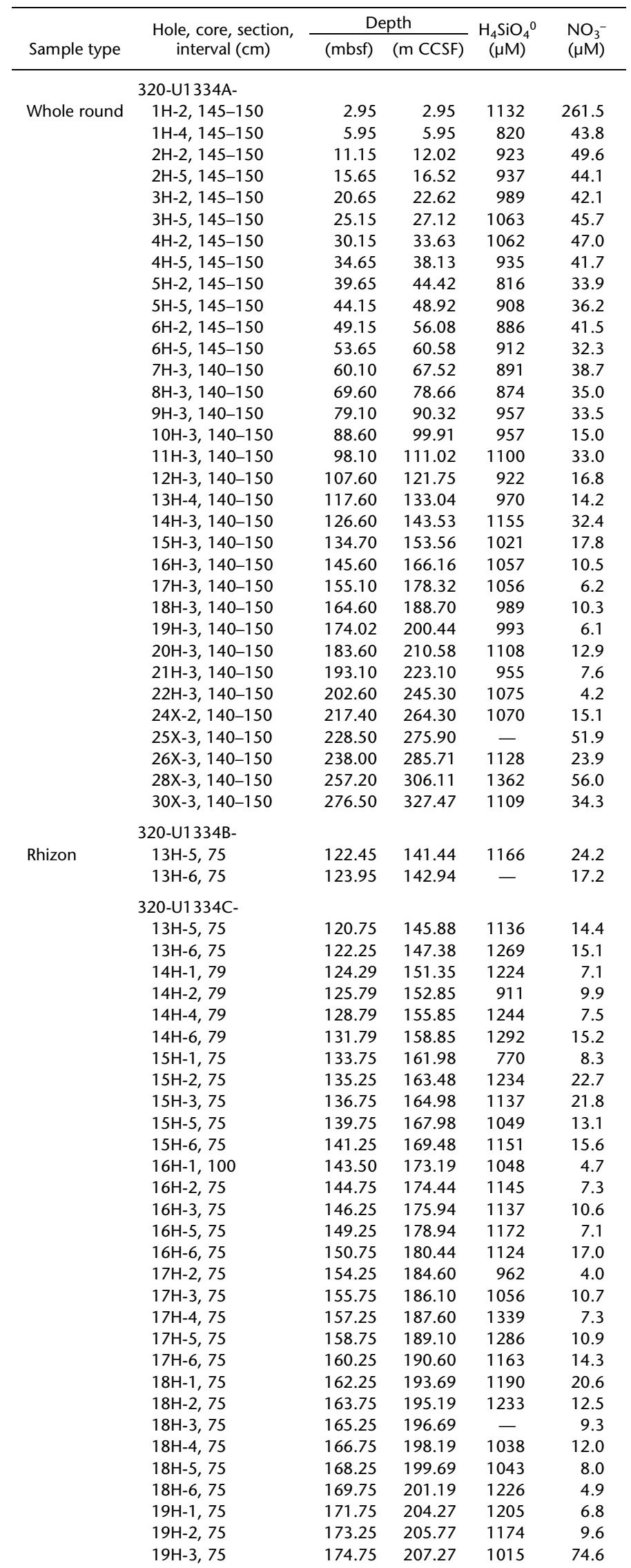


Table T6 (continued).

\begin{tabular}{|c|c|c|c|c|c|}
\hline \multirow[b]{2}{*}{ Sample type } & \multirow{2}{*}{$\begin{array}{l}\text { Hole, core, section, } \\
\text { interval }(\mathrm{cm})\end{array}$} & \multicolumn{2}{|c|}{ Depth } & \multirow{2}{*}{$\begin{array}{c}\mathrm{H}_{4} \mathrm{SiO}_{4}{ }^{0} \\
(\mu \mathrm{M})\end{array}$} & \multirow{2}{*}{$\begin{array}{l}\mathrm{NO}_{3}^{-} \\
(\mu \mathrm{M})\end{array}$} \\
\hline & & (mbsf) & (m CCSF) & & \\
\hline & $19 \mathrm{H}-4,75$ & 176.25 & 208.77 & - & 68.0 \\
\hline & $19 \mathrm{H}-5,75$ & 177.75 & 210.27 & 1077 & 24.2 \\
\hline & $19 \mathrm{H}-6,75$ & 179.25 & 211.77 & 1031 & 9.1 \\
\hline & $20 \mathrm{H}-1,75$ & 181.25 & 215.02 & 1250 & 13.3 \\
\hline & $20 \mathrm{H}-2,75$ & 182.75 & 216.52 & 1271 & 6.4 \\
\hline & $20 \mathrm{H}-4,75$ & 185.75 & 219.52 & 1254 & 12.8 \\
\hline & $20 \mathrm{H}-5,75$ & 187.25 & 221.02 & 1116 & 13.8 \\
\hline & $20 \mathrm{H}-6,75$ & 188.75 & 222.52 & 1015 & 3.9 \\
\hline & $21 \mathrm{H}-1,75$ & 190.75 & 224.95 & 1011 & 1.8 \\
\hline & $21 \mathrm{H}-2,75$ & 192.25 & 226.45 & 1058 & 5.6 \\
\hline & $21 \mathrm{H}-3,75$ & 193.75 & 227.95 & 946 & 1.7 \\
\hline & $21 \mathrm{H}-5,75$ & 196.75 & 230.95 & 858 & 9.1 \\
\hline & $21 \mathrm{H}-6,75$ & 198.25 & 232.45 & 1165 & 4.1 \\
\hline & $22 \mathrm{H}-1,75$ & 200.25 & 236.05 & 1182 & 5.2 \\
\hline & $22 \mathrm{H}-2,75$ & 201.75 & 237.55 & 1126 & 9.7 \\
\hline & $22 \mathrm{H}-3,75$ & 203.25 & 239.05 & 1175 & 9.0 \\
\hline & $22 \mathrm{H}-4,75$ & 204.75 & 240.55 & 1182 & 0.0 \\
\hline & $22 \mathrm{H}-5,75$ & 206.25 & 242.05 & 1096 & 7.0 \\
\hline & $22 \mathrm{H}-6,75$ & 207.75 & 243.55 & 1209 & 8.1 \\
\hline & $23 X-1,75$ & 209.75 & 250.68 & 1100 & 7.5 \\
\hline & $23 X-2,75$ & 211.25 & 252.18 & 1045 & 33.2 \\
\hline & $23 X-3,75$ & 212.75 & 253.68 & 987 & 11.7 \\
\hline
\end{tabular}

$-=$ no data. 
Table T7. Pore water concentrations, Site U1335.

\begin{tabular}{|c|c|c|c|c|c|}
\hline \multirow[b]{2}{*}{ Sample type } & \multirow{2}{*}{$\begin{array}{l}\text { Hole, core, section, } \\
\text { interval }(\mathrm{cm})\end{array}$} & \multicolumn{2}{|c|}{ Depth } & \multirow{2}{*}{$\begin{array}{l}\mathrm{H}_{4} \mathrm{SiO}_{4}{ }^{0} \\
(\mu \mathrm{M})\end{array}$} & \multirow{2}{*}{$\begin{array}{l}\mathrm{NO}_{3} \\
(\mu \mathrm{M})\end{array}$} \\
\hline & & (mbsf) & (m CCSF) & & \\
\hline & 320-U1335A- & & & & \\
\hline \multirow{48}{*}{ Whole round } & $1 \mathrm{H}-2,145-150$ & 2.95 & 3.09 & 983 & 58.3 \\
\hline & $1 \mathrm{H}-4,145-150$ & 5.95 & 6.09 & 802 & 46.6 \\
\hline & $2 \mathrm{H}-2,145-150$ & 11.85 & 12.86 & 927 & 152.8 \\
\hline & $2 \mathrm{H}-5,145-150$ & 16.35 & 17.36 & 1081 & 32.1 \\
\hline & $3 \mathrm{H}-2,145-150$ & 21.35 & 22.66 & 974 & 16.9 \\
\hline & $3 \mathrm{H}-5,145-150$ & 25.85 & 27.16 & 916 & 18.0 \\
\hline & $4 \mathrm{H}-2,145-150$ & 30.85 & 33.89 & 1076 & 14.0 \\
\hline & $4 \mathrm{H}-5,145-150$ & 35.35 & 38.39 & 1330 & - \\
\hline & $5 \mathrm{H}-2,145-150$ & 40.35 & 45.52 & 1069 & 12.9 \\
\hline & $6 \mathrm{H}-5,145-150$ & 54.35 & 60.34 & 1223 & 3.9 \\
\hline & $7 \mathrm{H}-3,140-150$ & 60.80 & 67.89 & 1050 & 0.8 \\
\hline & $8 \mathrm{H}-3,140-150$ & 70.30 & 79.49 & 988 & 1.6 \\
\hline & $9 \mathrm{H}-3,145-150$ & 78.58 & 89.94 & 911 & 3.9 \\
\hline & $10 \mathrm{H}-3,145-150$ & 89.35 & 100.40 & 870 & 14.8 \\
\hline & $11 \mathrm{H}-2,140-150$ & 97.30 & 110.16 & 936 & 9.7 \\
\hline & $12 \mathrm{H}-3,140-150$ & 108.30 & 122.39 & 821 & 6.2 \\
\hline & $13 \mathrm{H}-3,140-150$ & 117.80 & 131.04 & 878 & 6.3 \\
\hline & $14 \mathrm{H}-3,140-150$ & 127.30 & 142.60 & 882 & 6.7 \\
\hline & $15 \mathrm{H}-3,140-150$ & 136.30 & 152.65 & 899 & 6.1 \\
\hline & $16 \mathrm{H}-3,140-150$ & 146.30 & 162.65 & 819 & 2.4 \\
\hline & $17 \mathrm{H}-3,140-150$ & 155.80 & 175.80 & 886 & 5.1 \\
\hline & $18 \mathrm{H}-3,140-150$ & 165.30 & 185.77 & 1090 & 4.3 \\
\hline & $19 \mathrm{H}-3,140-150$ & 174.80 & 196.11 & 969 & 8.4 \\
\hline & $20 \mathrm{H}-3,140-150$ & 184.30 & 207.03 & 989 & 1.3 \\
\hline & $21 \mathrm{H}-3,140-150$ & 193.80 & 217.92 & 921 & 1.8 \\
\hline & $22 \mathrm{H}-3,140-150$ & 203.30 & 229.28 & 917 & 1.4 \\
\hline & $23 \mathrm{H}-3,140-150$ & 212.80 & 241.22 & 953 & 2.5 \\
\hline & $24 \mathrm{H}-3,140-150$ & 222.30 & 253.93 & 978 & 2.5 \\
\hline & $25 \mathrm{H}-3,140-150$ & 231.80 & 264.57 & 846 & 0.0 \\
\hline & $26 \mathrm{H}-3,140-150$ & 241.30 & 275.94 & 913 & 1.4 \\
\hline & $27 \mathrm{H}-3,140-150$ & 250.80 & 287.74 & 957 & 1.6 \\
\hline & $28 \mathrm{H}-3,140-150$ & 260.30 & 298.08 & 928 & 0.0 \\
\hline & $29 \mathrm{H}-3,140-150$ & 269.80 & 309.29 & 974 & 8.5 \\
\hline & $30 \mathrm{H}-3,140-150$ & 279.30 & 319.86 & 1003 & 11.7 \\
\hline & $31 \mathrm{H}-3,140-150$ & 288.80 & 331.43 & 943 & 5.5 \\
\hline & $32 \mathrm{H}-3,140-150$ & 298.30 & 342.26 & 969 & 8.4 \\
\hline & $33 \mathrm{H}-3,140-150$ & 307.80 & 353.54 & 946 & 7.9 \\
\hline & $34 \mathrm{H}-3,140-150$ & 315.90 & 364.32 & 1037 & 6.5 \\
\hline & $39 X-3,140-150$ & 365.10 & 417.10 & 1146 & 4.9 \\
\hline & $40 X-3,140-150$ & 374.60 & 426.60 & 913 & 9.6 \\
\hline & $41 X-3,140-150$ & 384.10 & 436.10 & 831 & 10.8 \\
\hline & $42 X-3,140-150$ & 393.60 & 445.60 & 1222 & 20.0 \\
\hline & $43 X-3,140-150$ & 403.20 & 455.20 & 1113 & 23.1 \\
\hline & $44 X-3,140-150$ & 412.80 & 464.80 & 989 & 33.8 \\
\hline & 320-U1335B- & & & & \\
\hline & $36 \mathrm{H}-3,140-150$ & 330.80 & 382.65 & 939 & 12.8 \\
\hline & $37 \mathrm{H}-2,140-150$ & 338.70 & 392.95 & 958 & 4.2 \\
\hline & $39 \mathrm{H}-3,140-150$ & 357.20 & 411.45 & 970 & 5.8 \\
\hline
\end{tabular}

$-=$ no data. 
Table T8. Pore water concentrations, Site U1336.

\begin{tabular}{ccrrr}
\hline Sample type & $\begin{array}{c}\text { Core, section, } \\
\text { interval (cm) }\end{array}$ & $\begin{array}{c}\text { Depth } \\
(\mathrm{mbsf})\end{array}$ & $\begin{array}{c}\mathrm{H}_{4} \mathrm{SiO}_{4}{ }^{0} \\
(\mu \mathrm{M})\end{array}$ & $\begin{array}{c}\mathrm{NO}_{3}{ }^{-} \\
(\mu \mathrm{M})\end{array}$ \\
\hline \multirow{5}{*}{ Whole round } & $\begin{array}{c}320-\mathrm{U} 1336 \mathrm{~B}- \\
2 \mathrm{H}-2,145-150\end{array}$ & 4.75 & 933 & 75.4 \\
& $2 \mathrm{H}-5,145-150$ & 9.25 & 924 & 76.2 \\
& $3 \mathrm{H}-2,145-150$ & 14.25 & 1093 & 63.3 \\
& $3 \mathrm{H}-5,145-150$ & 18.75 & 857 & 49.3 \\
& $4 \mathrm{H}-2,145-150$ & 23.75 & 918 & 46.2 \\
& $4 \mathrm{H}-5,145-150$ & 28.25 & 967 & 46.6 \\
& $5 \mathrm{H}-2,145-150$ & 33.25 & 844 & 40.7 \\
$5 \mathrm{H}-5,145-150$ & 37.75 & 1023 & 39.1 \\
$6 \mathrm{H}-2,145-150$ & 42.75 & 935 & 37.3 \\
$6 \mathrm{H}-5,145-150$ & 47.25 & 951 & - \\
$7 \mathrm{H}-3,145-150$ & 53.75 & 985 & 29.4 \\
$8 \mathrm{H}-3,140-150$ & 63.20 & 868 & 30.5 \\
$9 \mathrm{H}-3,140-150$ & 72.70 & 954 & 22.1 \\
$10 \mathrm{H}-4,140-150$ & 83.70 & 935 & 26.5 \\
$11 \mathrm{H}-4,140-150$ & 93.20 & 759 & 10.2 \\
$12 \mathrm{H}-4,140-150$ & 102.70 & 928 & 7.8 \\
$13 \mathrm{H}-4,140-150$ & 112.20 & 1001 & 6.0 \\
$14 \mathrm{H}-4,140-150$ & 121.70 & 810 & 22.3 \\
$15 \mathrm{H}-4,140-150$ & 131.20 & 861 & 8.9 \\
$18 \mathrm{H}-4,140-150$ & 151.30 & 909 & 12.9 \\
$19 \mathrm{H}-3,140-150$ & 159.30 & 854 & 10.7 \\
$2 \mathrm{H}-3,140-150$ & 168.80 & 1067 & 7.4 \\
\hline
\end{tabular}

$-=$ no data.

Table T9. Pore water concentrations, Site U1337. (Continued on next page.)

\begin{tabular}{|c|c|c|c|c|}
\hline Sample type & $\begin{array}{l}\text { Core, section, } \\
\text { interval }(\mathrm{cm})\end{array}$ & $\begin{array}{l}\text { Depth } \\
\text { (mbsf) }\end{array}$ & $\begin{array}{c}\mathrm{H}_{4} \mathrm{SiO}_{4}{ }^{0} \\
(\mu \mathrm{M})\end{array}$ & $\begin{array}{l}\mathrm{NO}_{3} \\
(\mu \mathrm{M})\end{array}$ \\
\hline \multirow{34}{*}{ Whole round } & 321-U1337A- & & & \\
\hline & $2 \mathrm{H}-2,145-150$ & 8.45 & 970 & 14.6 \\
\hline & $2 \mathrm{H}-5,145-150$ & 12.95 & 871 & 4.9 \\
\hline & $3 \mathrm{H}-2,145-150$ & 17.95 & 1019 & 4.8 \\
\hline & $3 \mathrm{H}-5,145-150$ & 22.45 & 956 & 30.7 \\
\hline & $4 \mathrm{H}-2,145-150$ & 27.45 & 1204 & 2.6 \\
\hline & $4 \mathrm{H}-5,145-150$ & 31.95 & - & 16.4 \\
\hline & $5 \mathrm{H}-2,145-150$ & 36.95 & 1200 & 5.5 \\
\hline & $5 \mathrm{H}-5,145-150$ & 41.45 & 972 & 5.5 \\
\hline & $6 \mathrm{H}-2,145-150$ & 46.45 & 1092 & 3.3 \\
\hline & $6 \mathrm{H}-5,145-150$ & 50.95 & 1048 & 5.8 \\
\hline & 7H-3, 145-150 & 57.45 & 1065 & 7.5 \\
\hline & $8 \mathrm{H}-3,145-150$ & 66.95 & 1152 & 6.9 \\
\hline & $9 \mathrm{H}-3,145-150$ & 76.45 & 1152 & 11.6 \\
\hline & $10 \mathrm{H}-3,145-150$ & 85.95 & 1181 & 4.6 \\
\hline & $11 \mathrm{H}-3,145-150$ & 95.45 & 1096 & 1.9 \\
\hline & $12 \mathrm{H}-3,145-150$ & 104.95 & 1255 & 1.9 \\
\hline & $13 \mathrm{H}-3,145-150$ & 114.45 & 1249 & 14.7 \\
\hline & $14 \mathrm{H}-3,150-155$ & 124.00 & 1183 & 13.6 \\
\hline & $15 \mathrm{H}-3,145-150$ & 133.45 & 1062 & 4.5 \\
\hline & $16 \mathrm{H}-3,145-150$ & 142.95 & 1169 & 4.9 \\
\hline & $17 \mathrm{H}-3,145-150$ & 152.45 & 1368 & 13.7 \\
\hline & $18 \mathrm{H}-3,147-152$ & 161.99 & 1286 & 21.2 \\
\hline & $19 \mathrm{H}-3,145-150$ & 171.45 & - & 6.3 \\
\hline & $20 \mathrm{H}-3,145-150$ & 180.95 & 1264 & 14.2 \\
\hline & $21 \mathrm{H}-3,145-150$ & 190.45 & 1479 & 30.6 \\
\hline & $23 X-3,145-150$ & 208.75 & 1178 & 10.7 \\
\hline & $24 X-2,145-150$ & 216.85 & 1374 & 5.6 \\
\hline & $25 X-3,145-150$ & 227.85 & 1272 & 11.7 \\
\hline & $26 X-3,145-150$ & 237.45 & 1224 & 3.1 \\
\hline & $28 X-2,140-150$ & 254.60 & 1443 & 9.0 \\
\hline & $29 X-2,140-150$ & 264.20 & 1209 & 14.5 \\
\hline & $31 X-3,140-150$ & 284.90 & 1374 & 23.3 \\
\hline & $32 X-3,140-150$ & 294.60 & 1512 & 3.7 \\
\hline
\end{tabular}


Table T9 (continued).

\begin{tabular}{|c|c|c|c|c|}
\hline Sample type & $\begin{array}{l}\text { Core, section, } \\
\text { interval }(\mathrm{cm})\end{array}$ & $\begin{array}{l}\text { Depth } \\
\text { (mbsf) }\end{array}$ & $\begin{array}{c}\mathrm{H}_{4} \mathrm{SiO}_{4}{ }^{0} \\
(\mu \mathrm{M})\end{array}$ & $\begin{array}{l}\mathrm{NO}_{3} \\
(\mu \mathrm{M})\end{array}$ \\
\hline & $33 X-3,140-150$ & 304.10 & 1357 & 10.9 \\
\hline & $34 X-3,140-150$ & 313.70 & 1451 & 8.4 \\
\hline & $35 X-3,140-150$ & 323.30 & 1792 & 12.8 \\
\hline & $36 X-3,140-150$ & 332.90 & 1462 & 8.8 \\
\hline & $37 X-3,140-150$ & 342.40 & 1556 & 11.7 \\
\hline & $38 X-2,140-150$ & 350.50 & 1428 & 6.5 \\
\hline & $39 X-3,140-150$ & 361.60 & 1393 & 16.0 \\
\hline & $40 X-3,140-150$ & 371.20 & 1425 & 6.8 \\
\hline & $44 X-3,140-150$ & 409.30 & 1965 & 15.1 \\
\hline & $45 X-3,140-150$ & 418.90 & 1367 & 44.7 \\
\hline & $46 X-3,140-150$ & 428.40 & 1357 & 64.1 \\
\hline \multirow[t]{27}{*}{ Rhizon } & $2 \mathrm{H}-1,145-150$ & 6.95 & 1258 & 20.7 \\
\hline & $2 \mathrm{H}-2,140-145$ & 8.40 & 1134 & - \\
\hline & $2 \mathrm{H}-5,140-145$ & 12.90 & 1104 & - \\
\hline & $2 \mathrm{H}-6,145-150$ & 14.45 & 1003 & 4.4 \\
\hline & $3 \mathrm{H}-1,145-150$ & 16.45 & 1016 & 7.0 \\
\hline & $3 \mathrm{H}-6,145-150$ & 23.95 & 1299 & 7.8 \\
\hline & $4 \mathrm{H}-1,145-150$ & 25.95 & 1030 & 2.2 \\
\hline & $4 \mathrm{H}-2,140-145$ & 27.40 & 1103 & 27.7 \\
\hline & $4 \mathrm{H}-5,140-145$ & 31.90 & 1006 & - \\
\hline & $4 \mathrm{H}-6,145-150$ & 33.45 & 1078 & 4.5 \\
\hline & $5 \mathrm{H}-1,145-150$ & 35.45 & - & 1.8 \\
\hline & $5 \mathrm{H}-2,140-145$ & 36.90 & 1174 & 8.9 \\
\hline & $5 \mathrm{H}-6,145-150$ & 42.95 & 1156 & 7.5 \\
\hline & $6 \mathrm{H}-1,145-150$ & 44.95 & 1174 & 4.5 \\
\hline & $6 \mathrm{H}-2,140-145$ & 46.40 & 1120 & 6.5 \\
\hline & $6 \mathrm{H}-5,140-145$ & 50.90 & 1098 & - \\
\hline & $6 \mathrm{H}-6,145-150$ & 52.45 & 1128 & 11.5 \\
\hline & 7H-1, 145-150 & 54.45 & 1184 & 15.2 \\
\hline & 7H-5, 145-150 & 60.45 & 983 & 28.4 \\
\hline & $7 \mathrm{H}-7,53-58$ & 62.53 & 1175 & 8.3 \\
\hline & $9 \mathrm{H}-1,145-150$ & 73.45 & 1159 & 6.1 \\
\hline & $9 \mathrm{H}-6,145-150$ & 80.95 & 1052 & 3.7 \\
\hline & $10 \mathrm{H}-1,145-150$ & 82.95 & 1244 & 5.6 \\
\hline & $10 \mathrm{H}-3,145-150$ & 85.95 & - & 3.1 \\
\hline & $10 \mathrm{H}-5,145-150$ & 88.95 & 1176 & 5.9 \\
\hline & $10 \mathrm{H}-7,26-31$ & 90.76 & 1243 & 3.3 \\
\hline & $11 \mathrm{H}-6,145-150$ & 99.95 & 1249 & 2.5 \\
\hline
\end{tabular}

$-=$ no data. 
Table T10. Pore water concentrations, Site U1338. (Continued on next page.)

\begin{tabular}{|c|c|c|c|c|c|}
\hline \multirow[b]{2}{*}{ Sample type } & \multirow{2}{*}{$\begin{array}{l}\text { Hole, core, section, } \\
\text { interval }(\mathrm{cm})\end{array}$} & \multicolumn{2}{|c|}{ Depth } & \multirow{2}{*}{$\begin{array}{l}\mathrm{H}_{4} \mathrm{SiO}_{4}{ }^{0} \\
\quad(\mu \mathrm{M})\end{array}$} & \multirow{2}{*}{$\begin{array}{l}\mathrm{NO}_{3} \\
(\mu \mathrm{M})\end{array}$} \\
\hline & & (mbsf) & $(\mathrm{m}$ CCSF) & & \\
\hline \multirow{44}{*}{ Whole round } & 321-U1338A- & & & & \\
\hline & $1 \mathrm{H}-1,145-150$ & 1.40 & 1.49 & 832 & 20.2 \\
\hline & $2 \mathrm{H}-2,145-150$ & 5.65 & 7.13 & 795 & 10.6 \\
\hline & $2 \mathrm{H}-5,145-150$ & 10.15 & 11.63 & 738 & 7.0 \\
\hline & $3 \mathrm{H}-2,145-150$ & 15.15 & 17.38 & 802 & 8.1 \\
\hline & $3 \mathrm{H}-5,145-150$ & 19.65 & 21.88 & 848 & 9.6 \\
\hline & $4 \mathrm{H}-2,145-150$ & 24.65 & 28.30 & 853 & 20.8 \\
\hline & $4 \mathrm{H}-5,145-150$ & 29.15 & 32.80 & 878 & 8.6 \\
\hline & $5 \mathrm{H}-2,145-150$ & 34.15 & 39.90 & 819 & 7.9 \\
\hline & $5 \mathrm{H}-5,145-150$ & 38.65 & 44.40 & 923 & 7.2 \\
\hline & $6 \mathrm{H}-2,145-150$ & 43.65 & 49.20 & 934 & 8.3 \\
\hline & $6 \mathrm{H}-5,145-150$ & 48.15 & 53.70 & 929 & 10.8 \\
\hline & $7 \mathrm{H}-3,145-150$ & 54.65 & 60.90 & 1199 & 38.0 \\
\hline & $8 \mathrm{H}-3,145-150$ & 64.15 & 72.20 & 1032 & 38.2 \\
\hline & $9 \mathrm{H}-3,145-150$ & 73.65 & 83.10 & 1087 & 6.7 \\
\hline & $10 \mathrm{H}-3,145-150$ & 83.15 & 93.40 & 1149 & 24.4 \\
\hline & $11 \mathrm{H}-3,145-150$ & 92.65 & 103.70 & 1215 & 7.7 \\
\hline & $12 \mathrm{H}-3,145-150$ & 102.15 & 114.50 & 1245 & 7.0 \\
\hline & $13 \mathrm{H}-3,145-150$ & 111.65 & 125.40 & 1474 & 8.8 \\
\hline & $14 \mathrm{H}-3,145-150$ & 121.15 & 135.10 & 1483 & 27.7 \\
\hline & $15 \mathrm{H}-3,145-150$ & 130.65 & 145.70 & 1400 & 56.0 \\
\hline & $16 \mathrm{H}-3,145-150$ & 140.15 & 156.20 & 1197 & 19.6 \\
\hline & $17 \mathrm{H}-3,145-150$ & 149.65 & 167.70 & 1151 & 21.7 \\
\hline & $18 \mathrm{H}-3,145-150$ & 159.15 & 178.40 & 1384 & 14.2 \\
\hline & $19 \mathrm{H}-3,145-150$ & 168.65 & 188.80 & 1275 & 14.9 \\
\hline & $20 \mathrm{H}-3,145-150$ & 178.15 & 198.90 & 1222 & 17.2 \\
\hline & $21 \mathrm{H}-3,145-150$ & 187.65 & 209.70 & 1459 & 13.8 \\
\hline & $22 \mathrm{H}-3,145-150$ & 197.15 & 220.50 & 1419 & 37.0 \\
\hline & $23 \mathrm{H}-3,145-150$ & 206.65 & 231.30 & 1420 & 20.5 \\
\hline & $24 \mathrm{H}-3,145-150$ & 216.15 & 242.60 & 1385 & 19.1 \\
\hline & $25 \mathrm{H}-3,146-151$ & 225.68 & 253.20 & 1441 & 48.3 \\
\hline & $26 \mathrm{H}-3,145-150$ & 235.15 & 263.10 & 1423 & 44.6 \\
\hline & $27 X-3,140-150$ & 244.60 & 272.40 & 1528 & 76.2 \\
\hline & $29 X-3,140-150$ & 263.80 & 293.10 & 1391 & 22.3 \\
\hline & $32 X-3,140-150$ & 292.50 & 380.00 & 1469 & 9.9 \\
\hline & $33 X-3,140-150$ & 302.10 & 392.40 & 1557 & 35.6 \\
\hline & $34 X-3,140-150$ & 311.70 & 425.10 & 1283 & 24.3 \\
\hline & $38 X-3,140-150$ & 349.90 & 450.80 & - & 57.3 \\
\hline & $29 X-3,140-150$ & 359.20 & 315.90 & - & 198.8 \\
\hline & $41 X-3,140-150$ & 378.40 & 358.30 & 1341 & 53.5 \\
\hline & $42 X-3,140-150$ & 388.00 & 434.30 & 1525 & 21.0 \\
\hline & $44 X-3,140-150$ & 405.70 & 450.80 & 1411 & 80.9 \\
\hline & $\begin{array}{l}321-\mathrm{U} 1338 \mathrm{~B}- \\
36 \mathrm{H}-3,140-150\end{array}$ & 325.30 & 434.30 & 1299 & 24.8 \\
\hline & 321-U1338A- & & & & \\
\hline \multirow[t]{23}{*}{ Rhizon } & $1 \mathrm{H}-2,100-105$ & 2.50 & 2.54 & 954 & 6.9 \\
\hline & $2 \mathrm{H}-1,145-150$ & 4.15 & 5.63 & 1132 & 31.1 \\
\hline & $2 \mathrm{H}-6,145-150$ & 11.65 & 13.13 & 1105 & 6.7 \\
\hline & $3 \mathrm{H}-1,145-150$ & 13.65 & 15.88 & 893 & 11.1 \\
\hline & $3 \mathrm{H}-6,145-150$ & 21.15 & 23.38 & 1205 & 11.3 \\
\hline & $4 \mathrm{H}-1,145-150$ & 23.15 & 26.77 & 1167 & 6.6 \\
\hline & $4 \mathrm{H}-6,145-150$ & 30.65 & 34.27 & 1160 & 4.4 \\
\hline & $5 \mathrm{H}-6,145-150$ & 40.15 & 45.85 & 939 & 9.6 \\
\hline & $6 \mathrm{H}-6,145-150$ & 49.65 & 55.21 & 1155 & 4.4 \\
\hline & 7H-1, 145-150 & 51.65 & 57.86 & 1160 & 7.3 \\
\hline & $7 \mathrm{H}-5,145-150$ & 57.65 & 63.86 & 1213 & 7.8 \\
\hline & $7 \mathrm{H}-7,71-76$ & 59.91 & 66.12 & 1187 & 14.1 \\
\hline & $8 \mathrm{H}-1,145-150$ & 61.15 & 69.23 & 1200 & 9.0 \\
\hline & $8 \mathrm{H}-5,145-150$ & 67.15 & 75.23 & 1243 & 9.2 \\
\hline & $8 \mathrm{H}-7,68-73$ & 69.38 & 77.46 & 1254 & 32.0 \\
\hline & $9 \mathrm{H}-1,145-150$ & 70.65 & 80.10 & 1054 & 12.8 \\
\hline & $9 \mathrm{H}-5,145-150$ & 76.65 & 86.10 & 1128 & 10.3 \\
\hline & $9 \mathrm{H}-7,53-58$ & 78.73 & 88.18 & 1016 & 8.2 \\
\hline & $10 \mathrm{H}-1,145-150$ & 80.15 & 90.38 & 1233 & 6.7 \\
\hline & $10 \mathrm{H}-5,145-150$ & 86.15 & 96.38 & - & 6.0 \\
\hline & $11 \mathrm{H}-4,145-150$ & 94.15 & 105.80 & 1086 & 11.4 \\
\hline & $12 \mathrm{H}-1,145-150$ & 99.15 & 111.50 & 1116 & 8.7 \\
\hline & $12 \mathrm{H}-5,145-150$ & 105.15 & 117.50 & 1086 & 6.6 \\
\hline
\end{tabular}


Table T10 (continued).

\begin{tabular}{|c|c|c|c|c|c|}
\hline \multirow[b]{2}{*}{ Sample type } & \multirow{2}{*}{$\begin{array}{l}\text { Hole, core, section, } \\
\text { interval }(\mathrm{cm})\end{array}$} & \multicolumn{2}{|c|}{ Depth } & \multirow{2}{*}{$\begin{array}{c}\mathrm{H}_{4} \mathrm{SiO}_{4}{ }^{0} \\
(\mu \mathrm{M})\end{array}$} & \multirow{2}{*}{$\begin{array}{l}\mathrm{NO}_{3}- \\
(\mu \mathrm{M})\end{array}$} \\
\hline & & (mbsf) & (m CCSF) & & \\
\hline & $12 \mathrm{H}-7,72-77$ & 107.42 & 119.77 & 1196 & 8.8 \\
\hline \multicolumn{6}{|c|}{ 321-U1338B- } \\
\hline & $14 \mathrm{H}-4,90-95$ & 127.00 & 140.05 & 1224 & 2.9 \\
\hline & $14 \mathrm{H}-4,100-105$ & 127.10 & 140.15 & 1257 & 14.4 \\
\hline & $14 \mathrm{H}-4,120-125$ & 127.30 & 140.35 & - & 3.4 \\
\hline & $14 \mathrm{H}-4,130-135$ & 127.40 & 140.45 & 1185 & 8.1 \\
\hline & $14 \mathrm{H}-4,140-145$ & 127.50 & 140.55 & 1212 & 1.2 \\
\hline & $14 \mathrm{H}-5,10-15$ & 127.70 & 140.75 & 1128 & 1.7 \\
\hline & $14 \mathrm{H}-5,20-25$ & 127.80 & 140.85 & 1073 & 6.8 \\
\hline & $14 \mathrm{H}-5,30-35$ & 127.90 & 140.95 & 1222 & 2.6 \\
\hline & $14 \mathrm{H}-5,40-45$ & 128.00 & 141.05 & 1160 & 5.0 \\
\hline & $14 \mathrm{H}-5,50-55$ & 128.10 & 141.15 & 1208 & 6.3 \\
\hline & $14 \mathrm{H}-5,60-65$ & 128.20 & 141.25 & 1111 & 19.0 \\
\hline & $14 \mathrm{H}-5,70-75$ & 128.30 & 141.35 & 1105 & 2.6 \\
\hline & $14 \mathrm{H}-5,80-85$ & 128.40 & 141.45 & 1158 & 2.5 \\
\hline & $14 \mathrm{H}-5,90-95$ & 128.50 & 141.55 & 877 & 5.6 \\
\hline & $14 \mathrm{H}-5,100-105$ & 128.60 & 141.65 & 1216 & 3.7 \\
\hline & $14 \mathrm{H}-5,110-115$ & 128.70 & 141.75 & 1162 & 1.4 \\
\hline & $14 \mathrm{H}-5,120-125$ & 128.80 & 141.85 & 1157 & 2.0 \\
\hline & $14 \mathrm{H}-5,130-135$ & 128.90 & 141.95 & 1203 & 5.5 \\
\hline & $14 \mathrm{H}-5,140-145$ & 129.00 & 142.05 & 986 & 5.9 \\
\hline & $14 \mathrm{H}-6,10-15$ & 129.20 & 142.25 & 1046 & 7.8 \\
\hline & $14 \mathrm{H}-6,30-35$ & 129.40 & 142.45 & 1120 & 13.1 \\
\hline & $14 \mathrm{H}-6,40-45$ & 129.50 & 142.55 & 1206 & 3.3 \\
\hline & $14 \mathrm{H}-6,50-55$ & 129.60 & 142.65 & 1193 & 3.4 \\
\hline & $14 \mathrm{H}-6,60-65$ & 129.70 & 142.75 & 1229 & 2.1 \\
\hline & $14 \mathrm{H}-6,70-75$ & 129.80 & 142.85 & 970 & 9.3 \\
\hline & $14 \mathrm{H}-6,80-85$ & 129.90 & 142.95 & 1147 & 5.6 \\
\hline
\end{tabular}

$-=$ no data. 